\title{
ASPECTOS ECONÔMICOS DA PRODUÇÃO E DO RISCO NOS SISTEMAS AGROFLORESTAIS E NOS SISTEMAS TRADICIONAIS DE PRODUÇÂO AGRÍCOLA EM TOMÉ-AÇU, PARÁ - 2001 A 2003'
}

\author{
Luiz Benedito Varela² e Antônio Cordeiro de Santana ${ }^{3}$
}

\begin{abstract}
RESUMO - Neste artigo, analisaram-se os fatores determinantes da produção dinâmica dos sistemas agroflorestais (SAF) e dos sistemas tradicionais de produção agrícola (ST), sob condições de risco, em pequenas e médias unidades produtivas nipo-brasileiras localizadas no Município de Tomé-Açu, Pará, no período de 2001 a 2003. Os resultados indicaram que todos os fatores, exceto a mão-de-obra contratada e as máquinas e equipamentos, afetam diretamente o Valor Bruto da Produção (VBP) dos SAF e dos ST; a variável dummy apresentou diferença cumulativa a menor no VBP dos SAF, de um ano para outro. A função de risco estimada apontou que os SAF apresentaram menor risco que os ST, evidenciando-se que a aplicação de insumos era fonte de redução de risco, mas a tecnologia adotada precisa ser adequada, pois se apresenta como fator de aumento de risco nos dois sistemas. Além disso, a dummy indicou que os SAF exibiram menor nível de risco que os ST. Nesse contexto, os resultados deixaram claro, ainda, que os produtores nipo-brasileiros eram avessos ao risco.
\end{abstract}

Palavras-chave: VBP, função de produção dinâmica, e sustentabilidade.

\section{ECONOMIC ASPECTS OF PRODUCTION UNDER RISK CONDITIONS IN AGROFORESTRY SYSTEMS AND TRADITIONAL AGRICULTURAL SYSTEMS IN TOMÉ-AÇU, PARÁ - 2001 TO 2003}

\begin{abstract}
This article analyzes the determinants of the dynamic production inputs of the agroforestry systems (SAF) and traditional agricultural systems (ST) under risk conditions, in small and medium farms in Tome-Açu, Pará, by Brazilian-Japanese producers from 2001 to 2003. The dynamic regression model results showed that all the inputs, except labor and machinery, have positive impacts on the current VBP of the two systems analyzed. Furthermore, the dummy variable shows a minor accumulative difference of the SAF's VBP in comparison with ST'S VBP, from one period (year) to another. The estimated risk function indicates that the use of fertilizer and pesticides reduce the risk level of the two systems, but the current technology needs to be adequate to other production inputs, since it appears as a factor of increased risk to the two systems. In addition, the dummy variable indicates that the SAF presents a lower level of risk than the ST. The results indicate that the Brazilian-Japanese farmers were .risk averse.
\end{abstract}

Keywords: VBP, dynamic production function and sustainability.

\footnotetext{
${ }^{1}$ Recebido em 16.08.2007 e aceito para publicação em 26.01.2009.

${ }^{2}$ UNAMA, Av. Alcindo Cacela, 287, Bloco E, $4^{\circ}$ Andar, Núcleo de Pesquisa em Socioeconomia. CEP 66060-902 Belém, Pará.E-mail: <varela@unama.br>.

${ }^{3}$ UFRA, Av. Tancredo Neves, 2501, CEP 66.077-530 Belém, Pará. E-mail: <santana@ nautilus.com.br>.
} 


\section{INTRODUÇÃO}

Desde meados do século passado, a agricultura brasileira vem experimentando mudanças nos métodos de produção e na produtividade, especialmente com a revolução verde. No final da década de 1970, os modelos de monocultura, predominantemente adotados no Centro-Sul, Sudeste e, em menor escala, no Norte do país, apresentavam instabilidade na produção e preços, devido aos riscos relativos a mercado, problemas fitossanitários e quebra de safra.

Algumas culturas de exportação priorizadas pelo II Plano Nacional de Desenvolvimento (II PND) foram exemplos disso. Os produtores de pimenta-do-reino (Piper nigrum), seringueira (Hevea brasiliensis), café (Coffea arabica) e cacau (Theobroma cacao) sofreram com a queda dos preços no mercado internacional, que se acentuou nos anos de 1980, contribuindo para desorganizar a produção e desestimular os agricultores. Além disso, a escassez e encarecimento do crédito agrícola, por conta da elevação dos níveis de inflação, limitaram os investimentos às culturas de ciclo longo, prejudicando a expansão desses cultivos.

Os problemas relacionados aos cultivos de exportação levaram os agricultores a buscar alternativas como a pecuária e a agricultura de subsistência. Contudo, essas alternativas agravaram os problemas ambientais, posto que os modelos de pecuária extensiva (grandes projetos) e de agricultura de subsistência, esta de caráter migratório ou nômade, intensificaram os desmatamentos na Amazônia (MENDES, 1997).

$\mathrm{Na}$ tentativa de superar esses problemas, os produtores buscam o estabelecimento de um modelo de produção sustentável do ponto de vista ambiental, econômico e social, e já se observa, em várias regiões, o inicio da gradativa substituição da prática do monocultivo tradicional por um modelo de produção que combina dois ou mais cultivos e criatórios ao mesmo tempo, de forma organizada. Esse processo de mudança de práticas agrícolas ocorreu em várias regiões do país a partir dos anos 1970, notadamente na Bahia (Una), no Acre, Amazonas, Rondônia (Ouro Preto), no Pará (Monte Alegre, Acará e Tomé-Açu) e em outras localidades da Amazônia.
A introdução organizada desse novo sistema de produção com diferentes combinações de cultivos e criatórios, sempre com a presença de uma espécie florestal - conhecidos como sistemas agroflorestais (SAF) vem-se consolidando de forma gradativa em ToméAçu e outras regiões, ao lado dos sistemas de produção chamados de tradicionais, de forma sustentável (HOMMA, 1998; BAENA e FALESI, 1999).

Todavia, algumas questões econômicas preocupam os pesquisadores e produtores na decisão sobre a adoção dos SAF em relação aos sistemas tradicionais: a) os fatores determinantes da produção; b) as fontes de risco desses sistemas; a escala espacial e de tempo da produção; e c) o monitoramento da sustentabilidade desse sistema não só através de indicadores ambientais e de sustentabilidade econômica e social. A viabilidade econômica dos SAF também depende, de certa forma, do papel relevante que esse tipo de sistema desempenha no "sequestro" de carbono (EMBRAPA, 2000).

\subsection{Definição do Problema}

O fundamento da economicidade constitui componente essencial no processo de escolha e decisão do agricultor no processo de adoção de sistemas de produção considerados preservacionistas, ou que envolvam o manejo e uso adequados da floresta e do solo, como é o caso dos SAF.

O problema é que muitas questões de fundo econômico dos SAFainda continuam dependentes de informação, pesquisa e estudos para a consecução de respostas científicas consistentes, tanto na esfera da produção, quanto no que diz respeito ao risco do empreendimento (YAMADA, 1999).

No âmbito econômico da produção e do risco, alguns pontos relacionados ao desempenho dos SAFs e ST (monocultivos tradicionais) necessitam de resposta. Dentre eles, os seguintes:

1. Quais os fatores determinantes da produção nos sistemas agroflorestais (SAF) e sistemas tradicionais ou monocultivos (ST)?

2. Quais os fatores determinantes do risco nos SAF e nos sistemas tradicionais de produção (ST)?

3. Os produtores rurais que adotam os SAF são aversos ou neutros em relação ao risco $^{4}$ ?

4 Embora não seja objeto desta pesquisa, cujo período se restringe a um "corte" de três anos no tempo (2001 a 2003), há que se considerar no estudo econômico dos SAF o risco da recuperação do capital investido no longo prazo (20 anos, em média), muito embora muitos produtores nipo-brasileiros, pela sua cultura e formação, apropriem os benefícios ambientais (solo, sequestro de carbono e efeito-estufa), além do efeito-poupança representado pela manutenção das espécies florestais. Nos SAF, mesmo após o período próprio de corte, alguns produtores mantêm "reservas florestais" a título de "poupança" para seus descendentes.

R. Árvore, Viçosa-MG, v.33, n.1, p.151-160, 2009

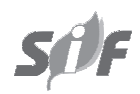




\subsection{Justificativa e Objetivos}

Os trabalhos de pesquisa (MARQUES et al., 1993; WALKER, 1994; YAMADA, 1999) e as experiências dos produtores geraram um considerável volume de informações de cunho técnico (biológico e ambiental), evidenciando as vantagens dos $\mathrm{SAF}$, dos pontos de vista ecológico e social. Todavia, no campo econômico os trabalhos sobre a viabilidade econômica dos SAF são poucos e de caráter estimativo. No entanto, a literatura brasileira sobre produção e risco na atividade agrícola e principalmente em SAF - não se desenvolveu de modo a contemplar a imensa heterogeneidade que existe na nossa ecologia. Na área da produção, alguns estudos recentes foram realizados por Castro et al. (2005) e Souza et al. (2005). Já na área de risco, pode-se citar o estudo de risco/retorno desenvolvido por Noce et al. (2005). Todavia, os referidos trabalhos enfocaram o setor florestal tradicional (ST) e não os SAF.

Preocupado com esse problema, as instituições de pesquisa vêm estimulando estudos que têm a finalidade de elucidar a influência da produção e dos riscos na alocação de recursos pelos agricultores (CRUZ, 1984), particularmente na área dos SAF, que representam um sistema com várias espécies produtivas e com períodos de safras distintos, mas sempre com uma base florestal.

A análise da produção dinâmica - metodologia apropriada para a análise econômica dos SAF pela sua característica multiestágio e multiperíodo - guarda estreita relação com os objetivos da pesquisa, à medida que representa investigação e geração de conhecimento e tecnologia sobre sistemas agroflorestais, objetivando a utilização eficiente dos recursos e o desenvolvimento sustentável (ANTLE,1983), num contexto dinâmico de produção.

Nesse contexto, o objetivo geral da pesquisa é o de analisar os fatores determinantes da produção dos sistemas agroflorestais e dos sistemas tradicionais praticados pelos produtores rurais do Município de Tomé-Açu, Estado do Pará, avaliando o risco desses sistemas, no período de 2001 a 2003. O trabalho tem os seguintes objetivos específicos: a) Identificar os determinantes da produção dinâmica nos sistemas agroflorestais e nos sistemas tradicionais de produção agrícola, estimando a função de produção dinâmica desses dois sistemas; e b) Estimar a função risco em cada um desses sistemas, identificando os fatores responsáveis pela variabilidade do produto, a fim de subsidiar o processo de decisão do produtor.

\section{ASPECTOS METODOLÓGICOS}

Esta seção trata inicialmente da área de estudo e apresenta os principais modelos de análise sobre a função de produção dinâmica e o risco associado aos dois sistemas de produção rural e trata da base de dados da pesquisa.

\section{1. Área de Estudo}

A área objeto deste estudo situa-se na microrregião de Tomé-Açu, Pará, mais precisamente no Município de Tomé-Açu, cuja sede se localiza nas margens do rio Acará, distante cerca de $93 \mathrm{~km}$ de sua foz no rio Guamá. Nessa região, distante $280 \mathrm{~km}$ de Belém por via rodoviária (PA-140) e $270 \mathrm{~km}$ pelos rios Acará e Guamá, existem várias propriedades rurais que desenvolvem sistemas agrícolas tradicionais ao lado de sistemas agroflorestais, cuja expansão vem predominando nos últimos anos (HOMMA,1998).

Os solos predominantes da região são os oxissolos, apresentando níveis baixos de pH e fertilidade limitada. Observou-se nas áreas de topografia plana o Latossolo Amarelo em suas fases texturais variando de média a argilosa e, nas de topografia ondulada, é comum a ocorrência do Concrecionário Laterítico (FALESI et al., 1994). O clima da área é do tipo Ami, da classificação de Köppen, com temperatura média anual de $27,9{ }^{\circ} \mathrm{C}$ e chuvas com um total anual em torno de $2.500 \mathrm{~mm}$, com distribuição irregular durante os meses, definindo duas estações, uma bastante chuvosa (de novembro a junho), e outra menos chuvosa (de julho a outubro), quando ocorrem totais mensais inferiores a $50 \mathrm{~mm}$, o que causa considerável deficiência hídrica (RODRIGUES e BAENA, 1974). Localizado entre os paralelos de $2^{0}$ $5^{\prime}$ e $3^{0} 15^{\prime}$ ao sul e os meridianos $48^{\circ} 35^{\prime}$ e $47^{\circ} 55^{\prime}$ a oeste da cidade de Belém, o município foi colonizado em boa parte por migrantes japoneses, experimentou as duas fases históricas de ocupação agrícola: a primeira, nas décadas de 1940 a 1970, com a predominância da monocultura da pimenta-do-reino e cacau; e a fase atual iniciada com a introdução dos sistemas agroflorestais (HOMMA, 1998; YAMADA, 1999).

\subsection{Modelos de Análise}

\subsubsection{Função de Produção Dinâmica}

A forma funcional utilizada é o de uma função de produção dinâmica, ajustada para captar os efeitos defasados do valor da produção passada sobre a

R. Árvore, Viçosa-MG, v.33, n.1, p.151-160, 2009 
produção atual, através de um modelo dinâmico de regressão múltipla (SANTANA, 2003).

O modelo dinâmico de regressão especificado para determinação do valor bruto da produção e do risco nos sistemas agroflorestais consiste na função tipo transcendental de dois estádios utilizada por Santana (1992), sem as inadequações da função clássica identificadas por Just e Pope (1979).

No estágio I, o valor bruto da produção (VBP) é determinado, conforme Santana (1992), pelos seguintes fatores: área cultivada (AA), insumos (IN), tecnologia (TC) e pelos efeitos do valor bruto da produção do ano anterior $\left(\mathrm{VBP}_{\mathrm{t}-1}\right)$ (inseridos na parte Cobb-Douglas da função) e pela mão-de-obra contratada (MO), máquinas e equipamentos (ME) e pela variável dummy (DU) (incorporados na parte exponencial da função).

O modelo de análise foi montado com dois tipos de variáveis dummy, representadas por $\mathrm{DU}_{1}$ e $\mathrm{DU}_{2}$. A variável dummy $\mathrm{DU}_{1}$ foi inserida na função para captar possíveis diferenças entre a produção dos SAF (com valor 1) e dos Sistemas Tradicionais (com valor zero) e que eventualmente provoquem o deslocamento da função de produção. A dummy $\mathrm{DU}_{2}$ foi testada para captar eventuais diferenças de produção de um ano para outro, entre os dois sistemas, sendo atribuído valor zero para o primeiro ano e valor 1 para o segundo ano da série de tempo, em cada um dos dois sistemas. Referida função é representada por um modelo dinâmico autor-regressivo ${ }^{5}$ com a seguinte configuração (SANTANA, 1992, 2003):

$$
\begin{aligned}
\mathrm{VBP}_{\mathrm{t}}= & \alpha_{0}\left(\mathrm{AA}_{\mathrm{t}}\right)^{\alpha 1}\left(\mathrm{IN}_{\mathrm{t}}\right)^{\alpha 2}\left(\mathrm{TC}_{\mathrm{t}}\right)^{\alpha 3}\left(\mathrm{VBP}_{\mathrm{t}-1}\right)^{\alpha 4} \cdot \exp \cdot\left[\alpha_{5}(\mathrm{DU})\right. \\
& \left.+\alpha_{6}\left(\mathrm{MO}_{\mathrm{t}}\right)+\alpha_{7}\left(\mathrm{ME}_{\mathrm{t}}\right)\right]+\mathrm{v}_{\mathrm{t}}
\end{aligned}
$$

logaritmando (1),

$$
\begin{gathered}
\log \mathrm{VBP}=\alpha_{0}+\alpha_{1} \log \mathrm{AA}+\alpha_{2} \log \mathrm{IN}+\alpha_{3} \log \mathrm{TC}+\alpha_{4} \log \\
\mathrm{VBP}_{\mathrm{t}-1}+\alpha_{5} \mathrm{DU}+\alpha_{6} \mathrm{MO}_{\mathrm{t}}+\alpha_{7} \mathrm{ME}_{\mathrm{t}}+\mathrm{v}_{\mathrm{t}}
\end{gathered}
$$

\subsubsection{Função de Risco}

A função risco é representada pelo estágio II da função de produção dinâmica. Nesse estágio, a variabilidade da produção ou elasticidade-risco é determinada pela função logarítmica do termo de erro da expressão anterior (SANTANA, 1992), ou seja: $\log \left(\mathrm{V}_{\mathrm{t}}\right)=\beta 0_{+} \beta_{1} \log \mathrm{AA}_{\mathrm{t}}+\boldsymbol{\beta}_{2} \log \mathrm{IN}_{\mathrm{t}+} \boldsymbol{\beta}_{3} \log \mathrm{TC} \mathrm{C}_{\mathrm{t}}+\boldsymbol{\beta}_{4} \log$

$\mathrm{VBP}_{\mathrm{t}-1}+\beta_{5} \mathrm{DU}_{\mathrm{t}}+\beta_{6} \mathrm{MO}_{\mathrm{t}}+\beta_{7} \mathrm{ME}_{\mathrm{t}}+\mathrm{u}_{\mathrm{t}}$

em que:

$\mathrm{VBP}_{\mathrm{t}}=$ valor bruto da produção agrícola, expresso em reais de dezembro de 2003 ;

$\mathrm{AA}_{\mathrm{t}}=$ área de terra efetivamente cultivada, em hectares;

$\mathrm{IN}_{\mathrm{t}}=$ valor dos adubos e defensivos utilizados, em reais de dezembro de 2003 ;

$\mathrm{TC}_{\mathrm{t}}=$ tecnologia, expressa em índice de produtividade relacionado à produtividade média do cultivo tradicional ou solteiro da região;

$\mathrm{VBP}_{\mathrm{t}-1}=$ valor bruto da produção agrícola do ano anterior, expresso em reais de dezembro de 2003;

$\mathrm{DU}_{\mathrm{t}}=$ variável dummy expressa por $\mathrm{DU}_{1}$ para captar diferença de produção entre SAF (1) e ST (0) ou por $\mathrm{DU}_{2}$ para testar diferenças de produção entre um ano (0) e o subsequente (1), em cada um dos dois sistemas de produção;

$\mathrm{MO}_{\mathrm{t}}=$ mão-de-obra contratada, expressa em homensdia (h/d) de trabalho;

$\mathrm{ME}_{\mathrm{t}}=$ valor das máquinas e equipamentos agrícolas, expresso em reais de dezembro de 2003;

$\alpha_{\mathrm{i}} \mathrm{e} \beta_{\mathrm{i}}=$ coeficientes das variáveis da função de produção dinâmica e da função risco, respectivamente; e

$v_{t}$ e $u_{t}=$ termos de erro aleatórios da função de produção e da função de risco, respectivamente.

\subsection{Base de Dados}

Os dados utilizados na análise do modelo são oriundos de duas fontes: a primeira, de natureza operacional, feita através de pesquisa direta de campo, com informações sobre valor bruto da produção (VBP), quantidade produzida (QT), área cultivada (AA), preço dos produtos (P), valor dos insumos (IN), valor das máquinas e equipamentos (ME), quantidade da mãode-obra (MO) utilizada na produção e sobre a tecnologia (TC) de cultivo, além de informações sobre a comercialização dos produtos para venda e subsistência,

\footnotetext{
${ }^{5} \mathrm{O}$ modelo de função de produção dinâmica não se refere à defasagem distribuída dos insumos e fatores de produção, a exemplo do modelo que foi utilizado por Mesquita (1996) para medir o impacto de mudança tecnológica em sistemas tradicionais de produção. Neste estudo, considera-se o VBP ${ }_{t}$ como variável monetária independente, defasada de um período, como variável explicativa do VBP atual e do Risco.
}

R. Árvore, Viçosa-MG, v.33, n.1, p.151-160, 2009 
levantados na CAMTA-Cooperativa Agrícola Mista de Tomé-Açu; e a segunda, de cunho institucional, relativamente às informações sobre a atuação dos órgãos como a EMBRAPA, UFRA, Empresa de Assistência Técnica e extensão Rural do Pará (EMATER-PA), entre outros.

A pesquisa de campo utilizou formulários ajustados, aplicados sobre uma amostra de 36 unidades rurais conduzida intencionalmente, sendo 18 unidades produtivas do tipo SAF e 18 do tipo tradicional (ST), esta última caracterizada pela exploração de cultivos solteiros. Amaioria das unidades produtivas de SAF e ST existentes em ToméAçu possui tamanho de até 20 ha, consideradas pequenas ou médias propriedades em relação às unidades com áreas bem maiores e que praticam outras atividades como a pecuária extensiva ou semiextensiva.

As dificuldades de obtenção das informações de uma série de tempo maior, em nível de campo, limitaram a amostra dos dados coletados aos períodos agrícolas de 2001, 2002 e 2003, num total de 108 observações (= 36 unidades x 3 anos), incluindo SAF e ST.

Em razão disso, por decisão metodológica dos autores do trabalho, o modelo de análise aplicado combina dados de série de tempo com informações dos sistemas agroflorestais e tradicionais (cross section), levantados em cada um desses períodos. As variáveis utilizadas foram previamente testadas e selecionadas pelos autores do estudo, com base critérios estatísticos. Assim, as variáveis especificadas com valor monetário (VBP, IN e ME) foram atualizadas a preços de dezembro de 2003 pelo Índice Geral de Preços, disponibilidade interna (IGP-DI) da Fundação Getúlio Vargas (FGV); já as variáveis quantificadas fisicamente (QT, AA, MO) permaneceram com as respectivas unidades originais, com exceção da tecnologia (TC) que foi medida através de um índice construído a partir do referencial da produtividade média ${ }^{6}$ de cada sistema de produção tradicional praticado na região (cultivo solteiro).

\section{RESULTADOS E DISCUSSÃO}

\subsection{Determinantes do VBP na unidade produtiva}

A análise do comportamento do VBP como função de produção dinâmica dos insumos e recursos utilizados pelos produtores, em nível de unidade produtiva, resultou os resultados constantes da Tabela 1 .

Tabela 1 - Regressão estimada do VBP como função dos insumos e fatores de produção, em nível de propriedade. ToméAçu, Pará 2001-2003

Table 1 - VBP estimated regression as a function of inputs and production factors, at property level. Tomé-Açu, Pará 2001-2003

Variável Dependente: LOG(VBP)

Método: Mínimos Quadrados Ordinários

Data: 26/01/06 Hora: 21:52

Observações: 72

Corrigido pelo teste White de Heterocedasticidade

\begin{tabular}{|c|c|c|c|c|}
\hline Variavel & Coeficiente & Erro-Padrão & Estatistica-t & Probabilid. \\
\hline $\mathrm{C}$ & 1.321744 & 0,547585 & 2.413770 & 0,0187 \\
\hline LOG(AA) & 0,107602 & 0,086714 & 1.240886 & 0,1992 \\
\hline LOG(IN) & 0,063153 & 0,041724 & 1.513600 & 0,1351 \\
\hline LOG(TC) & 0,069385 & 0,042504 & 1.632437 & 0,1075 \\
\hline $\operatorname{LOG}\left(\mathrm{VBP}_{1}\right)$ & 0,835324 & 0,072382 & 11.54044 & 0,0000 \\
\hline MO & $-4.16 \mathrm{E}-06$ & $1.04 \mathrm{E}-05$ & $-0,401610$ & 0,6893 \\
\hline ME & $-4.12 \mathrm{E}-07$ & $7.35 \mathrm{E}-07$ & $-0,560666$ & 0,5770 \\
\hline $\mathrm{DU}_{2}$ & $-0,263535$ & 0,063888 & -4.124976 & 0,0001 \\
\hline $\mathrm{R}$-quadrado $\left(\mathrm{R}^{2}\right)$ & 0,918903 & Media variavel dependente & & 11.06497 \\
\hline $\mathrm{R}^{2}$ Ajustado & 0,910033 & S.D. variavel dependente & & 0,897248 \\
\hline S.E. da regressão & 0,269125 & Criterio inf. Akaike & & 0,317160 \\
\hline S.quadrado residuo & 4.635421 & Criterio Schwarz & & 0,570123 \\
\hline Log probabilidade & -3.417763 & Estatística-F & & 103.5965 \\
\hline Durbin-Watson (d) & 2.117597 & Prob. estatística-F & & 0,000000 \\
\hline
\end{tabular}

Fonte: Elaboração do próprio autor, 2006.

${ }^{6} \mathrm{O}$ índice de produtividade foi construído supondo-se a produtividade média de qualquer cultivo solteiro igual a 1 . A partir daí, a maior ou menor densidade de plantas de cada cultivar por hectare em SAF - dependendo do espaçamento, de sua idade e do seu estágio vegetativo (plantio, tratos cultural ou colheita) - determina a produtividade quase sempre menor do que 1, por hectare, porque no SAF a densidade de plantas de cada cultivar é menor do que nos ST, embora a densidade de todos os cultivares nos SAF seja maior do que nos ST. 
Os valores do teste $t$ (Student) da Tabela 1 indicam a significância estatística do intercepto e dos coeficientes do valor bruto da produção passada $\left(\mathrm{VBP}_{\mathrm{t}-1}\right)$ e da variável dummy $\left(\mathrm{DU}_{2}\right){ }^{7}$ em nível de $5 \%$ de probabilidade de erro. Os coeficientes da tecnologia (TC), dos insumos (IN) e da área cultivada (AA) mostraram-se significantes a $15 \%$ e $20 \%$ de probabilidade de erro. Isso demonstra a influência positiva dessas variáveis sobre o valor bruto da produção dos SAF e ST, em nível de propriedade.

Os parâmetros da mão-de-obra (MO) e das máquinas e equipamentos (ME) mostram-se estatisticamente não significantes, indicando que não exercem influência sobre o VBP, em nível de propriedade.

O R $\mathrm{R}^{2}$ mostra que $91,8 \%$ das variações no VBP dos SAF são explicadas pela mudança nas variáveis do modelo, enquanto os valores e a significância da estatística $F$ indicam a adequação do modelo para o estudo proposto.

Deve-se mencionar que a amostra analisada apresentou problema de heterocedasticidade, conforme os resultados a seguir discriminados, mas os valores foram corrigidos com a aplicação do método White. Ademais, no teste de correlação entre as variáveis explicativas os resultados do coeficiente de correlação simples ao quadrado entre os pares de variáveis $\left(\mathrm{r}^{2}\right)$ apresentaram-se inferiores ao coeficiente de determinação $\left(\mathrm{R}^{2}\right)$ da regressão, indicando ausência de problemas sérios de multicolinearidade no modelo.

Do ponto de vista econômico, os sinais dos coeficientes de elasticidades da regressão apresentaramse coerentes com o postulado da teoria da produção dinâmica, com exceção dos parâmetros mão-de-obra e máquinas e equipamentos, que se apresentaram negativos, indicando que esses fatores atuam no estágio irracional (estágio III) da produção.

O sinal do intercepto mostra que VBP dos SAF e ST são positivos mesmo na ausência dos insumos e fatores de produção, inclusive do VBP defasado de um período.

O coeficiente de elasticidade do VBP em relação à área cultivada no valor de 0,1076 indica que uma expansão de $100 \%$ na área de cultivo resulta em aumento de $10,7 \%$ no valor bruto da produção dos SAF e ST, ceteris paribus.

Relativamente aos insumos, a elasticidade da produção de 0,0631 indica que um aumento de $100 \%$ na aplicação de fertilizantes e defensivos resulta num aumento de $6,3 \%$ no VBP dos sistemas analisados, tudo o mais permanecendo constante.

No que concerne à tecnologia, o coeficiente de elasticidade da produção de 0,0693 evidencia que um aumento de $100 \%$ no uso desse fator implica crescimento de $6,9 \%$ no VBP dos SAF e dos sistemas tradicionais de produção, tudo o mais constante.

O coeficiente positivo de elasticidade/ajustamento do VBP atual em relação ao VBP defasado de um período $(0,8353)$ indicou que as decisões tomadas pelo produtor no passado $\left(\mathrm{VBP}_{1}\right)$ têm impacto significativo sobre o valor da produção atual.

Tais resultados são coerentes com os obtidos por Chavas et al. (1985) e Fawcett (1973), atestando que o processo de produção agrícola ocorre de maneira dinâmica, em que o tempo influencia decisivamente o nível de produção. As decisões tomadas no passado atuam sobre a produção física, o nível de uso dos insumos, a área cultivada, a mão-de-obra contratada e a posição dos preços do produto.

Nesse contexto, os resultados confirmaram as indicações obtidas por Antle (1983) de que no processo decisório da produção agrícola se observam dois tipos de dinâmica: a dinâmica do produto, representada pela influência do VBP do período anterior sobre o atual, e a dinâmica dos insumos, decorrente do efeito dos insumos aplicados no passado para obtenção da produção atual.

\subsection{Determinantes do risco ( $\log$ IRI) em unidade produtiva}

A análise do comportamento do nível de risco (Log IRI) em unidades produtivas com SAF e sistemas tradicionais, em função da variabilidade dos insumos e dos fatores dinâmicos aplicados na produção, apresentou os resultados constantes da Tabela 2 .

$7 \mathrm{Na}$ análise dessa função foi introduzida e testada inicialmente a dummy $\left(\mathbf{D U}_{\mathbf{1}}\right)$ para captar possíveis efeitos diferenciais entre o VBP das UPSAF (com valor 1) e UPST (com valor zero) num mesmo período de tempo t, mas o coeficiente dessa variável não se apresentou estatisticamente significante, indicando que inexiste diferença significativa entre o VBP dos dois sistemas. Por isso, testou-se a dummy $\left(\mathbf{D U}_{2}\right)$, para captar possíveis diferenças intertemporais do VBP entre os dois sistemas, obtendo a significância estatística dos resultados.

R. Árvore, Viçosa-MG, v.33, n.1, p.151-160, 2009

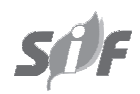


Tabela 2 - Regressão estimada do risco em função dos insumos e fatores de produção, em nível de propriedade. Tomé-AçuPará. 2001-2003

Table 2 - Risk estimated regression as a function of inputs and production levels at property level. Tomé-Açu-Pará 20012003

\begin{tabular}{|c|c|c|c|c|}
\hline $\begin{array}{l}\text { Variavel dependente } \\
\text { Método: Mínimos Q } \\
\text { Data: 27/01/06 } \\
\text { Observações: } 72 \\
\text { Corrigido pelo Méto }\end{array}$ & $\begin{array}{l}\text { OG IRI } \\
\text { drados Ordin } \\
21: 07 \\
\text { White de H }\end{array}$ & idade & & \\
\hline Variavel & Coeficiente & Erro-Padrão & Estatística-t & Probab. \\
\hline LOG(AA) & 0,022602 & 0,234311 & 0,096460 & 0,9234 \\
\hline LOG(IN) & $-0,212651$ & 0,088753 & -2.395983 & 0,0193 \\
\hline LOG(TC) & 0,177166 & 0,107921 & 1.641627 & 0,1053 \\
\hline $\mathrm{DU}_{2}$ & $-0,572690$ & 0,215398 & -2.658748 & 0,0098 \\
\hline $\mathrm{R}$-quadrado $\left(\mathrm{R}^{2}\right)$ & 0,162919 & Media variav & & -2.016095 \\
\hline $\mathrm{R}^{2}$ Ajustado & 0,125989 & S.D. variavel & & 1.065115 \\
\hline S.E. da regressão & 0,995761 & Criterio inf. $f$ & & 2.883333 \\
\hline S. quadrado residuo & 67.42466 & Criterio Schw & & 3.009814 \\
\hline Log probabilidade & -99.79998 & Estatística-F & & 4.411548 \\
\hline Durbin-Watson (d) & 1.772258 & Prob. estatís & & 0,006792 \\
\hline
\end{tabular}

Fonte: Elaboração do próprio autor, 2006.

Os resultados do teste $\boldsymbol{t}$ apresentados na Tabela 2 indicaram que os coeficientes das variáveis fertilizantes e defensivos (IN) e da variável dummy ( $\mathrm{DU}_{2}$ ) apresentaram-se estatisticamente significantes a $5 \%$ de probabilidade de erro, enquanto o parâmetro da tecnologia (TC) mostrou-se significante a $20 \%$. Isso indica a influência dessas variáveis sobre a variabilidade do produto (risco) dos SAF e dos ST, em nível de propriedade. O coeficiente da área cultivada (AA) não se apresentou significante, evidenciando que não representa fonte de risco para os sistemas analisados.

O valor do coeficiente de determinação $\left(R^{2}=0,1629\right)$ indicou que $16,3 \%$ das variações no risco da produção dos SAF e ST são explicadas pelas mudanças ocorridas na área cultivada, na aplicação de insumos, no uso da tecnologia, no emprego da mão-de-obra e nos efeitos sobre a variabilidade do produto captados pela dummy.

O valor da estatística $F=4,4115$ significante a $1 \%$ de probabilidade de erro demonstra que a equação estimada é adequada ao estudo do risco nos SAF e nos sistemas tradicionais de produção agrícola.

Os resultados da análise inicial indicaram a existência de heterocedasticidade - conforme os valores abaixo discriminados -, mas foram corrigidos pelo método White de heterocedasticidade. No entanto, a mostra apresentou problemas de multicolinearidade, a julgar pela comparação entre o quadrado dos coeficientes de correlação $\left(\mathrm{r}^{2}\right)$ dos pares da variáveis explicativas
$\mathrm{VBP}_{1}, \mathrm{MO}$ e $\mathrm{ME}$, que se apresentaram maiores que o coeficiente de determinação $\left(\mathrm{R}^{2}\right)$ da regressão múltipla de risco, tendo essas variáveis sido excluídas da análise.

A análise econômica indicou que os sinais dos coeficientes da regressão estimada apresentaram-se coerentes com a teoria do risco, no sentido de que cada variável pode contribuir para o aumento ou diminuição do risco do produto, dependendo do sinal positivo ou negativo do parâmetro de cada variável.

Nesse caso, os sinais negativos dos coeficientes dos insumos, da mão-de-obra e da dummy indicaram que o risco da produção dos SAF e dos ST diminuem a cada período de produção e, ou, à medida que se expande o uso desses insumos e fatores. O sinal positivo do parâmetro da tecnologia indicou que a expansão do uso desse fator, na forma atual, aumentou o risco dos sistemas de produção analisados.

A elasticidade de risco em relação aos insumos $(-0,212)$ mostrou que aumento de $100 \%$ na aplicação de fertilizantes e defensivos pode reduzir em $21,2 \%$ o risco dos SAF e ST, tudo o mais permanecendo constante.

Por sua vez, o coeficiente de elasticidade de risco da tecnologia, da ordem de 0,177 , indica que o aumento de $100 \%$ no uso desse fator resulta num crescimento de $17,7 \%$ no risco dos sistemas sob análise, ceteris paribus.

R. Árvore, Viçosa-MG, v.33, n.1, p.151-160, 2009 
A significância do coeficiente de elasticidade de risco da variável dummy, com valor de - 0,572, evidencia que os SAF apresentam menor nível de risco a cada período de produção, em comparação com os sistemas tradicionais, certamente em decorrência do maior efeito da produção de biomassa dos SAF sobre a produtividade do solo, ao longo do tempo.

Esses resultados, embora representativos apenas do período 2001-2003, evidenciam a preferência do produtor pelos SAF em relação aos ST, por apresentarem menor risco; o nível atual de risco, medido pelos coeficientes de elasticidade-risco dos fatores, pode ser inclusive reduzido com o aumento da aplicação dos insumos e com o reexame da tecnologia ora adotada, bem como da sua combinação com os demais fatores de produção.

A título de comparação, resultados de pesquisa recente sobre risco realizada em sistemas frutícolas tradicionais na Região Norte Fluminense, utilizando o método Monte Carlo, indicaram que os cultivos de manga (Mangifera indica) e goiaba (Psidium guyava) apresentaram maiores riscos em relação aos de maracujá (Passiflora edulis), graviola (Anona muricata) e outros, tendo sido apontados o nível de preço, a mão-de-obra contratada, os fertilizantes e a mecanização agrícola como determinantes da rentabilidade desses sistemas (PONCIANO et al., 2004)

Os estudos apresentados por Santos (1996), também utilizando o método Monte Carlo, mostraram a viabilidade econômica e o baixo risco do modelo de SAF constituído de arroz (Oriza sativa), banana (Musa sp), cacau e espécies florestais, praticado pelos pequenos produtores da Transamazônica, Pará. Na análise de cultivos individuais (ST), os resultados indicaram a inviabilidade econômica para a rizicultura.

\section{CONCLUSÕES E SUGESTÕES}

Com base nos resultados, algumas conclusões e sugestões podem servir de referência inicial à pesquisa e à orientação dos produtores dos sistemas agroflorestais e dos sistemas tradicionais de produção na região.

O conhecimento prévio dos fatores que influenciam o nível de produção e o risco dos SAF e ST pode, de alguma forma, evitar decisões que conduzam a inadequadas combinações de atividades e de fatores, considerando- se a dinâmica do tempo (ciclo vegetativo), do espaço da produção (unidade produtiva) e do meio ambiente com os quais os sistemas produtivos interagem, na busca pela otimização econômica da produção e da renda.

Os resultados da função de produção dinâmica em nível de unidade produtiva evidenciaram que todas as variáveis, exceto a mão-de-obra contratada e as máquinas e equipamentos, afetam diretamente o VBP dos SAF e ST. A exemplo do resultado obtido em nível de sistema, a variável dummy confirma, em nível de propriedade, a diferença cumulativa a menor do VBP dos SAF em relação à dos ST, de um ano para outro.

Tais resultados evidenciaram que, tanto nos sistemas agroflorestais quanto nos sistemas agrícolas tradicionais de produção praticados em Tomé-Açu, a utilização da mão-de-obra contratada e das máquinas e equipamentos deve ser racionalizada e mais bem combinada com os outros recursos e fatores de produção, a fim de aumentar o valor bruto da produção dos dois sistemas. O efeito de cada fator sobre o VBP pode ser medido pelas suas elasticidades parciais de produção. No entanto, a diferença a menor no VBP dos SAF em relação aos ST, de um período para outro, decorre do curto período analisado (2001 a 2003), época em que a maioria dos SAF pesquisados apresentava receita da produção dos intercultivos (cash crops), mas não contavam ainda com a receita da produção dos cultivos florestais (perenial crops).

A função risco estimada em nível de propriedade indicou que, enquanto a aplicação de insumos reduz o nível de risco, o produtor deve ser cauteloso no uso da tecnologia adotada, pois se apresenta como fator de aumento do risco nos SAF e ST, assim como ocorreu em nível de sistema. A variável dummy evidenciou também a diferença de risco entre os SAF e os ST, denotando que os SAF apresentam menor risco do que os sistemas tradicionais, repetindo o resultado apresentado em nível de sistema.

Uma análise comparativa entre os resultados da função de produção, da função risco e do retorno decrescente à escala de produção ${ }^{8}$ pode levantar o questionamento sobre as razões pelas quais o produtor nipo-brasileiro da região, que pratica uma área média de 20 ha, não expande sua produção. Nesse caso, embora a função de produção e o retorno à escala indiquem

\footnotetext{
${ }^{8} \mathrm{O}$ autor apresenta estudo sobre a rentabilidade econômica, retorno à escala e eficiência econômica em artigo apresentado em junho de 2006 na Universidade Federal Rural da Amazônia (UFRA); quanto ao retorno à escala, os resultados indicaram que os SAF pesquisados em Tomé-Açu apresentam área média de cultivo da ordem de 20 ha e operam com retornos decrescentes à escala.
} 
o aumento da produção, a função risco evidencia que a capacidade de gestão relacionada à tecnologia, a mão-de-obra e ao capital mostra-se inadequada, indicando que esses recursos devem ser adequadamente combinados com os demais fatores de produção. Nesse contexto, o produtor que não expande sua produção pode ser considerado avesso ao risco.

Diante dessa situação, a sugestão é de que o processo de expansão dos SAF e dos sistemas tradicionais deve ser precedido de um reexame pela pesquisa e pelo produtor sobre o planejamento e uso combinado dos fatores tecnologia, mão-de-obra e capital na atividade agrícola, de forma a ajustar a utilização desses recursos em combinação com os outros, a fim de buscar a eficiência econômica e reduzir o risco dos sistemas analisados.

A expansão da produção atual propiciaria condições de ajustar a utilização dos insumos e fatores de produção, o que teoricamente redundaria na redução do risco, dentro do que estabelece a hipótese risco-eficiência proposta por Antle (1983). Mais ainda, tal expansão, se planejada com base em estudo de mercado e suporte tecnológico, pode-se consolidar como um agronegócio que permitiria a conquista de maior fatia de mercado (market share) à produção, em substituição ao lucro, que passaria a ser objetivo estratégico de longo prazo, em razão do desempenho da rentabilidade econômica desses sistemas.

\section{REFERÊNCIAS}

ANTLE, J. M. Incorporating risk in production analysis. American Journal of Agricultural Economics, v.65, n.5, 1983.

BAENA, A. R. C.; FALESI, I..C. Avaliação do potencial químico e físico dos solos sob diversos sistemas de uso da terra na colônia agrícola de Tomé-Açu Estado do Para. Belém: Embrapa-CPATU, 1999. (Boletim de Pesquisa, 18).

BRIENZA JÜNIOR, S. Considerações biológicas e econômicas sobre um sistema de produção silvo-agrícola rotativo na região do Tapajós. Belém: Embrapa-CPATU, 1983, 22p. (Boletim de Pesquisa, 50).

COOPERATIVAAGRÍCOLA MISTA DE TOMÉAÇU - CAMTA. Relatórios de Campo: Ficha de cadastro de cooperado, 2001-2003. Quatro Bocas: 2004.
CASTRO, C. et al. Análise econômica do cultivo e extração do óleo esencial de Melalaeuca alternifolia Cheel. Revista Árvore, v.29, n.2, p.241-249, 2005.

CHAVAS, J.; KLIEBENSTEIN, J.; CRENSHAW, T. D. Modeling dynamic agricultural production response: The case of swine production.

American Journal of Agricultural Economics, v. 67, n.3, 1985.

COMISSÃO MUNDIAL SOBRE MEIO AMBIENTE E DESENVOLVIMENTO - CMMAD. Relatório Brundtand. Nosso futuro comum. 2.ed. Rio de Janeiro: FGV, 1991.

COUTINHO, L. As sete pragas da Amazônia. Revista Veja, 2005.

CRUZ, E. R. Risco em modelos de decisão na agricultura. Brasília: Embrapa, 1984.

CRUZ, E. R. Aspectos teóricos sobre incorporação de risco em modelos de decisão. In: CONTINI, E.; ALVES, E. Planejamento da propriedade agrícola: modelos de decisão. 2.ed. Brasília: Embrapa, 1986. p.237-260.

(Documentos, 7).

DILlon, J. L. Agricultura, pesquisa e probabilidade. Fortaleza: Universidade Federal do Ceará, 1976. 25p. (Série de Pesquisa, 13).

EMPRESA BRASILEIRA DE PESQUISA

AGROPECUÁRIA - EMBRAPA.

Reflorestamento de propriedades rurais para fins produtivos e ambientais: um guia para ações municipais e regionais. Brasília: 2000.

FAWCETT, R. H. Toward a dynamic production function. Journal of Agricultural Economics. v.24, p.543-55, 1973.

FERGUSON, C. E. The neoclassical theory of production and distribution. New York: Cambridge University Press, 1975.

HOMMA, A. K. O. Amazônia: meio ambiente e desenvolvimento agrícola. Brasília: Embrapa-SPI. Belém: Embrapa-CPATU, 1998.

R. Árvore, Viçosa-MG, v.33, n.1, p.151-160, 2009 
INSTITUTOBRASILEIRODE GEOGRAFIA E ESTATÍSTICA - IBGE. Indicadores de desenvolvimento sustentável: Brasil 2002. Rio de Janeiro: Departamento de Geociências, 2002.

\section{INTERNATIONAL COUNCIL FOR RESEARCH IN} AGROFORESTRY - ICRAF. Resources for agroforestry diagnosis and design. Kenia: ICRAF, 2000. 110p.

JUST, R. E.; POPE, R. Production function estimation and related risk considerations. American Journal of Agricultural Economics, v.61, n.2, p.276-84, 1979.

KNIGHT, F. H. Risk, uncertainty and profit. Boston: Houghton, Miffilin, 1921.

MacDICKEN, K. G.;VERGARA, N. T.

Agroforestry: classification and management. New York, Wiley-Interscience, 1990.

MARQUES, L. C. T.; YARED, J. A. G.; FERREIRA, C. A. P. Alternativa agroflorestal para pequenos produtores agrícolas, em áreas de terra firme no município de Santarém, Pará. Belém: Embrapa/CPATU, 1993.

MENDES, F. A. T. A sustentabilidade socioeconômica das áreas cacaueiras na Transamazônica: uma contribuição ao desenvolvimento regional. 1997. Tese (Doutorado) Escola Superior de Agrisultura Luiz de Queiroz, Piracicaba, 1997.

MESQUITA, T. C. Impacto da mudança tecnológica na produção agrícola: aproximação de uma análise dinâmica. Cadernos de Ciência e Tecnologia, v.13, n.2, p.159-173,1996.

NOCE, R. et al. Análise de risco e retorno do setor florestal: produtos da madeira. Revista Árvore, v.29, p.77-84, 2005.

PONCIANO, N.J. et al. Análise da viabilidade econômica e risco da fruticultura na Região Norte Fluminense. Revista de Economia e Sociologia Rural, v.42, n.4, 2004.

R. Árvore, Viçosa-MG, v.33, n.1, p.151-160, 2009
REIJNTES, C.; HAVERKORT B.; WATERSBAYERA, C. Agricultura para o Futuro: uma introdução à agricultura sustentável de baixo uso de insumos externos. Rio de Janeiro: ILEIA/ AS-PTA, 1994.

SANTANA, A. C. Analise econômica da produção agrícola sob condições de risco numa comunidade Amazônica. Revista de Economia e Sociologia Rural, v.30, n.2, p.159-170, 1992.

SANTANA, A. C.; TOURINHO, M. M. Notas sobre avaliação socioeconômica de sistemas agroflorestais. In: AUTOR. O agronegócio brasileiro: desafios e perspectivas. Local: Editora, 1997.

SAntAnA, A. C. Métodos quantitativos em economia: elementos e aplicações. Belém: Graphitte Editores, 2003.

SANTOS, J. C. Análise da rentabilidade, sob condições de risco, de um sistema agroflorestal adotado por pequenos produtores de cacau na região da transamazônica, Pará. 1996. 128f.

Dissertação (Mestrado) - Universidade Federal do Ceará, Fortaleza, 1996.

SOUZA, D. R. et al. Ciclo de corte econômico ótimo em floresta ombrófila densa de terra firme sob manejo florestal sustentável, Amazônia Oriental. Revista Arvore, v. 28, n.5, p.681-689, 2004.

WALKER, R. T.; HOMMA, A. K. O.; COUTO, A. J. Sistemas agroflorestais como processo evolutivo: o caso dos agricultores da rodovia Cuiabá-Santarém, no Estado do Pará. In: CONGRESSO BRASILEIRO SOBRE SISTEMAS AGROFLORESTAIS, 1., 1994, Porto Velho. Anais... Colombo: Embrapa-CNPF, 1994. p.29-42.

YAMADA, M. Japanese immigrant agroforestry in the Brazilian Amazon: A case study of sustainable rural development in the tropics. 1999. Tese (Doutorado em Economia Agrícola) - University of Florida, 1999. 